Pacific Journal of Mathematics

EQUIVALENCE OF DECOMPOSABLE AND 2-DECOMPOSABLE 


\title{
EQUIVALENCE OF DECOMPOSABLE AND 2-DECOMPOSABLE OPERATORS
}

\author{
MEHDI RADJABALIPOUR
}

\begin{abstract}
It is shown that every 2-decomposable operator (in the sense of $S$. Plafker) is decomposable (in the sense of $C$. Foias); this answers a question raised by Plafker.
\end{abstract}

Throughout the paper, $T$ is a bounded linear operator defined on a Banach space $Y$. An invariant subspace $X$ of $T$ is called a spectral maximal subspace of $T$ if $M \subseteq X$ for all invariant subspaces $M$ of $T$ for which $\sigma(T \mid M) \subseteq \sigma(T \mid X)$. The operator $T$ is called $n$ decomposable [10] ( $n$ a fixed integer greater than 1 ) if for every open covering $\left\{G_{1}, G_{2}, \cdots, G_{n}\right\}$ of $\sigma(T)$ there exist spectral maximal subspaces $X_{1}, X_{2}, \cdots, X_{n}$ of $T$ such that $\sigma\left(T \mid X_{i}\right) \subseteq \bar{G}_{i}(i=1,2, \cdots, n)$ and $Y=X_{1}+X_{2}+\cdots+X_{n} ; T$ is called decomposable [6] if it is $n$-decomposable for all $n \geqq 2$.

Plafker [10] asked whether every $n$-decomposable operator is decomposable; the question is answered by E. Albrecht and F.-H. Vasilescu [1] for $n \geqq 3$ in a general Banach space and by S. Frunză [7] for $n \geqq 2$ in a reflexive Banach space. Here we extend Frunză's result to a general Banach space by a shorter and simpler proof, and thus we completely solve Plafker's problem.

For a closed subset $F$ of $C$, we let $X_{T}(F)=\{x \in Y$ : there exists an analytic function $f_{x}: C \backslash F \rightarrow Y$ such that $\left.(z-T) f_{x}(z) \equiv x\right\}$. If $T$ is 2-decomposable, then $X_{T}(F)$ is a spectral subspace for all $F$, and every spectral maximal subspace of $T$ is of this form [10] (see also [4] for the case of decomposable operators). I. Colojoară and C. Foias [4, page 217] ask whether the restriction of a decomposable operator to every spectral maximal subspace is again decomposable; an operator whose restriction to every spectral maximal subspace is decomposable is called strongly decomposable by C. Apostol [2]. Obviously every strongly decomposable operator is decomposable and whether the converse is true is what Colojoară and Foiaș ask. I. Bacalu [3] shows that decomposable operators with nowhere dense spactra are strongly decomposable. The problem in the general case seems to be difficult and has been attacked by many authors; the following question is simpler and arises in a natural way.

Question 1. Does every decomposable operator $T$ satisfy the following condition (1):

$$
X_{T}(F) \cong X_{T}\left(\bar{G}_{1}\right)+X_{T}\left(\bar{G}_{2}\right)+\cdots+X_{T}\left(\bar{G}_{n}\right)
$$


for any closed set $F$ and any open covering $\left\{G_{1}, G_{2}, \cdots, G_{n}\right\}$ of $F$.

Decomposakle operators satisfying (an equivalent modification of) condition (1) are said to have almost localized spectra by Vasilescu [13], who also shows that the duals of such operators are again decomposable operators of the same type. Frunză [7], [8] shows that the dual of any 2-decomposable operator is decomposable and satisfies (1); hence in a reflexive Banach space all decomposable operators satisfy (1). We improve this result by showing that every decomposable operator (on any Banach space) satisfies (1).

We will make use of the following proposition due to Frunză [8, Proposition 1].

Proposition 1. Let $T$ be a 2-decomposable operator. Assume $X_{T}(F) \subseteq X_{T}\left(\bar{G}_{1}\right)+X_{T}\left(\bar{G}_{2}\right)$ for any closed set $F$ and any open covering $\left\{G_{1}, G_{2}\right\}$ of $F$. Then $T$ is a decomposable operator satisfying (1).

The following lemma is probably known to the experts; we include a proof for an easy reference. Recall that the operator $T$ is said to have the single-valued extension property if there exists no nonzero, $Y$-valued, analytic function $f$ such that $(z-T) f(z) \equiv 0$; it is known that every 2-decomposable operator $T$ has the singlevalued extension property and $\sigma\left(T \mid X_{T}(F)\right) \subseteq F \cap \sigma(T)$ for all closed sets $F$ [10] (see also [4]).

LEMMA 1. Let $T$ be a 2-decomposable operator and let $E$ be a closed subset of $C$. Let $\hat{Y}=Y / X_{T}(E), \hat{T}$ be the operator on $\hat{Y}$ induced by $T$, and let $\hat{x}$ in $\hat{Y}$ denote the image under the canonical mapping of $x \in Y$. Assume $\hat{x} \in X_{\hat{T}}(F)$ for some closed set $F$. Then $x \in$ $X_{T}(E \cup F)$.

Proof. Let $G_{1}$ be an open neighborhood of $E \cup F$ and let $G_{2}$ be another open set such that $\sigma(T) \subseteq G_{1} \cup G_{2}$ and $\bar{G}_{2} \cap(E \cup F)=\varnothing$. Let $x=x_{1}+x_{2}$, where $x_{i} \in X_{T}\left(\bar{G}_{i}\right)(i=1,2)$. Since $\hat{x}_{2}=\hat{x}-\hat{x}_{1}$, there exists and analytic function $g: C \backslash \bar{G}_{1} \rightarrow \hat{Y}$ such that $(z-\hat{T}) g(z) \equiv \widehat{x}_{2}$. Let $M=X_{T}\left(\bar{G}_{2} \cup E\right) / X_{T}(E)$. Since $M$ is a spectral maximal subspace of $\hat{T}$ containing $\widehat{x}_{2}$ [2, Proposition 1.3.2(3)], it follows that $g(z) \in M$ for all $z \notin \bar{G}_{1}$ [4, page 19]. (Note that, in view of [12, Theorem 2.1], $T$ has the single-valued extension property.) Now let $S: X_{T}\left(\bar{G}_{2}\right) \rightarrow M$ be the operator defined by $S y=\widehat{y}\left(y \in X_{T}\left(\bar{G}_{2}\right)\right)$. In the light of the Riesz decomposition theorem (applied to $T \mid X_{T}\left(\bar{G}_{2} \cup E\right)$ ), $S$ is bijective and $S\left(T \mid X_{T}\left(\bar{G}_{2}\right)\right)=(\widehat{T} \mid M) S$. Thus $(z-T)\left(S^{-1} g(z)\right) \equiv x$ for $z \notin \bar{G}_{1}$ and hence $x_{2} \in X_{T}\left(\bar{G}_{1}\right)$. Therefore $x \in X_{T}(\bar{G})$ for all neighborhoods $G$ of $E \cup F$ which implies that $x \in X_{T}(E \cup F)$.

Now we prove the main result of the paper. 
THEOREM 1. Every 2-decomposable operator $T$ is decomposable and satisfies condition (1), i.e., it has almost localized spectrum.

Proof. Let $F$ be a closed subset of the plane and let $\left\{G_{1}, G_{2}\right\}$ be an open covering of $F$. Let $E=\bar{G}_{1} \cap \bar{G}_{2}, \hat{Y}=Y / X_{T}(E)$, and let $\hat{T}$ be the operator on $\hat{Y}$ induced by $T$. Let $x \in X_{T}(F)$ and let $f: C \backslash F \rightarrow Y$ be an analytic function such that $(z-T) f(z) \equiv x$. Obviously $\hat{f}: C \backslash F \rightarrow \hat{Y}$ is analytic and $(z-\hat{T}) \hat{f}(z) \equiv \hat{x}$. (Here again $\hat{u}$ denotes the image of $u$ under the canoninal mapping from $Y$ onto $\hat{Y}$.) Since $\sigma(\hat{T}) \leqq C \backslash\left(G_{1} \cap G_{2}\right)[9$, Lemma 1], it follows that $\hat{f}$ has an analytic extension (denoted by the same symbol $\hat{f})$ to $(C \backslash F) \cup\left(G_{1} \cap G_{2}\right)$, which also preserves the identity $(z-\hat{T}) \hat{f}(z) \equiv \widehat{x}$.

Let $D_{1}$ be a Cauchy domain containing $F \backslash G_{2}$ and let $D_{2}$ be another one containing $F \backslash G_{1}$. Since $F \backslash G_{1}$ and $F \backslash G_{2}$ are disjoint, $D_{1}$ and $D_{2}$ can be chosen such that $\bar{D}_{1} \cap \bar{D}_{2}=\varnothing$. Now for $j=1,2$ define

$$
\xi_{j}=(2 \pi i)^{-1} \int_{+\partial D_{j}} \hat{f}(\lambda) d \lambda
$$

and

$$
g_{j}(z)=(2 \pi i)^{-1} \int_{+\partial D_{j}}(z-\lambda)^{-1} \widehat{f}(\lambda) d \lambda\left(z \notin D_{j}\right) .
$$

Obviously $\hat{x}=\xi_{1}+\xi_{2}$. Also,

$$
\begin{aligned}
(z-\hat{T}) g_{j}(z) & =(2 \pi i)^{-1} \int_{+\partial D_{j}}((z-\lambda)+(\lambda-\hat{T}))(z-\lambda)^{-1} \hat{f}(\lambda) d \lambda \\
& =(2 \pi i)^{-1} \int_{+\partial D_{j}} \hat{f}(\lambda) d \lambda+(2 \pi i)^{-1} \int_{+\partial D_{j}}(z-\lambda)^{-1} \hat{x} d \lambda=\xi_{j},
\end{aligned}
$$

for $z \notin D_{j}$ and $j=1$, 2. This shows that $\xi_{1} \in X_{\hat{T}}\left(\bar{D}_{1}\right)$, and since $D_{1}$ is an arbitrary Cauchy domain containing $F \backslash G_{2}$, it follows that $\xi_{1} \in X_{\hat{T}}\left(F \backslash G_{2}\right)$. Similarly $\xi_{2} \in X_{\hat{T}}\left(F \backslash G_{1}\right)$. Let $x_{j}$ be a vector in $Y$ such that $\xi_{j}=\hat{x}_{j}(j=1,2)$. By Lemma 1, $x_{j} \in X_{T}\left(\bar{G}_{j}\right)(j=1,2)$. Since $\hat{x}=\hat{x}_{1}+\hat{x}_{2}, \quad x=\left(x_{1}+u\right)+x_{2}$, where $u \in X_{T}(E)$; thus $x \in X_{T}\left(\bar{G}_{1}\right)+$ $X_{T}\left(\bar{G}_{2}\right)$. Now the rest of the proof follows from Proposition 1 .

Part (c) of the following corollary gives a new characterization of decomposable operators.

COROLLARY 1. The following assertions are equivalent.

(a) $T$ is decomposable.

(b) $T$ is 2-decomposable.

(c) For every closed set $F, X_{T}(F)$ is closed and $\sigma\left(T^{F}\right) \subseteq \sigma(T) \backslash F^{\circ}$, where $T^{F}$ denotes the operator induced by $T$ on $Y / X_{T}(F)$ and $F^{\circ}$ denotes the interior of $F$. 
Proof. First note that the operator $T$ in each case has the single-valued extension property (for case (c) see [11, Remark 2]). Now the equivalence of (a) and (b) follows from Theorem 1 and the equivalence of (b) and (c) follows from [9].

REMARK. J. Daughtry [5] defines a superinvariant subspace of $T$ to be a subspace $M$ invariant under all operators $A$ such that $(A T-T A) M \subseteq M$; he shows that an invariant subspace of a normal operator is superinvariant if and only if it is the range of a spectral projection. Note that if $T$ is a normal operator with the resolution of the identity $E_{T}$, then $X_{T}(F)=E_{T}(F) Y$ for all closed sets $F$. The following proposition shows that for any decomposable operator $T$ the subspaces $X_{T}(F)$ are superinvariant.

Proposition 2. Let $T$ be a decomposable operator and let $F$ be a closed subset of $C$. Then $X_{T}(F)$ is a superinvariant subspace of $T$.

Proof. Let $T_{F}=T \mid X_{T}(F)$. Let $x \in X_{T}(F)$ and let $f: C \backslash F \rightarrow Y$ be an analytic function such that $(T-\lambda) f(\lambda) \equiv x$. Let $A$ be an operator such that $(T A-A T) X_{T}(F) \subseteq X_{T}(F)$ and let

$$
g(\lambda)=A f(\lambda)-\left(T_{F}-\lambda\right)^{-1}(T A-A T) f(\lambda)(\lambda \notin F) .
$$

Since $\sigma\left(T_{F}\right) \subseteq F$ and $f(\lambda) \in X_{T}(F)$ for $\lambda \notin F$ [4, page 19], it follows that $g$ is a well-defined analytic function and $(T-\lambda) g(\lambda) \equiv A x$. Thus $A x \in X_{T}(F)$ and the proof is complete.

\section{REFERENCES}

1. E. Albrecht and F.-H. Vasilescu, On spectral capacities, Rev. Roum. Math. Pures Appl., 19 (1974), 701-705.

2. C. Apostol, Spectral decomposition and functional calculus, Rev. Roum. Math. Pures Appl., 13 (1968), 1481-1528.

3. I. Bacalu, On restrictions and quotients of decomposable operators, Rev. Roum. Math. Pures Appl., 18 (1973), 809-813.

4. I. Colojoarǎ and C. Foiaș, TheTheory of Generalized Spectral Operators, Gordon Breach, Science Publ., New York (1968).

5. J. Daughty, Superinvariant subspaces, (to appear).

6. C. Foiaș, Spectral maximal spaces and decomposable operators in Banach spaces, Arch. Math. (Basel), 14 (1963), 341-349.

7. S. Frunză, Spectral decomposition and duality, Illinois J. Math., (to appear).

8. — A new result of duality for spectral decompositions, (to appear).

9. A. A. Jafarian and F.-H. Vasilescu, A characterization of 2-decomposable operators, Rev. Roum. Math. Pures Appl., 19 (1974), 769-771.

10. S. Plafker, On decomposable operators, Proc. Amer. Math. Soc., 24 (1970), 215-216. 11. M. Radjabalipour, On subnormal operators, Trans. Amer. Math. Soc., 211 (1975), 377-389. 
12. F.-H. Vasilescu, Residual properties for closed operators on Frechet spaces, Illinois J. Math., 15 (1971), 377-386.

13. - On the residual decomposability in dual spaces, Rev. Roum. Pures. Appl., 16 (1971), 1573-1578.

Received August 2, 1976 and in revised form November 1, 1977. The research was done in an operator theory institute at the University of New Hampsphire.

REZA Shah Kabir University

P. O. BoX 444

BABOLSAR, IRAN 



\section{PACIFIC JOURNAL OF MATHEMATICS}

\section{EDITORS}

RICHARD ARENS (Managing Editor)

University of California

Los Angeles, California 90024

C. W. Curtis

University of Oregon

Eugene, OR 97403

C. C. MOORE

University of California

Berkeley, CA 94720

\section{J. DUGUNDJI}

Department of Mathematics University of Southern California Los Angeles, California 90007

R. Finn AND J. Milgram Stanford University Stanford, California 94305

\section{ASSOCIATE EDITORS}

E. F. BeCK ENBACH

B. H. NeUMaNN

F. WOLF

K. Yoshida

\section{SUPPORTING INSTITUTIONS}

UNIVERSITY OF BRITISH COLUMBIA CALIFORNIA INSTITUTE OF TECHNOLOGY UNIVERSITY OF CALIFORNIA MONTANA STATE UNIVERSITY UNIVERSITY OF NEVADA, RENO NEW MEXICO STATE UNIVERSITY OREGON STATE UNIVERSITY UNIVERSITY OF OREGON
UNIVERSITY OF SOUTHERN CALIFORNIA STANFORD UNIVERSITY UNIVERSITY OF HAWAII UNIVERSITY OF TOKYO UNIVERSITY OF UTAH WASHINGTON STATE UNIVERSITY UNIVERSITY OF WASHINGTON 


\section{Pacific Journal of Mathematics \\ Vol. 77, No. $1 \quad$ January, 1978}

Dan Amir, Chebyshev centers and uniform convexity ............... 1

Lawrence Wasson Baggett, Representations of the Mautner group. I ..... 7

George Benke, Trigonometric approximation theory in compact totally

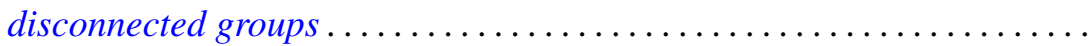

M. Bianchini, O. W. Paques and M. C. Zaine, On the strong compact-ported topology for spaces of holomorphic mappings ..................

Marilyn Breen, Sets with $(d-2)$-dimensional kernels

J. L. Brenner and Allen Kenneth Charnow, Free semigroups of $2 \times 2$

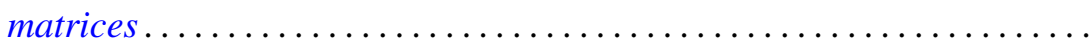

David Bressoud, A new family of partition identities .................

David Fleming Dawson, Summability of matrix transforms of stretchings

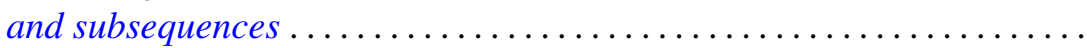

Harold George Diamond and Paul Erdôs, A measure of the nonmonotonicity

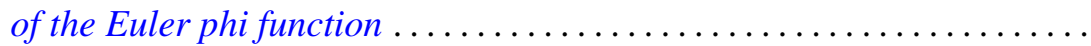

Gary Doyle Faulkner and Ronald Wesley Shonkwiler, Kernel dilation in reproducing kernel Hilbert space and its application to moment

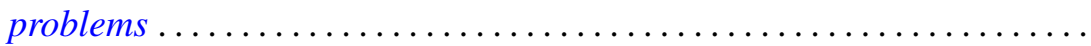

Jan Maksymilian Gronski, Classification of closed sets of attainability in the

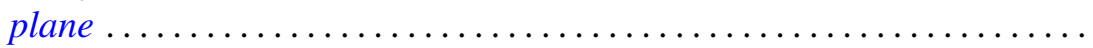

H. B. Hamilton and T. E. Nordahl, Semigroups whose lattice of congruences

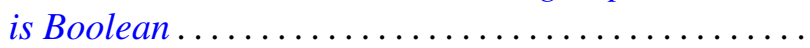

Harvey Bayard Keynes and D. Newton, Minimal $(G, \tau)$-extensions ...

Anthony To-Ming Lau, The Fourier-Stieltjes algebra of a topological

semigroup with involution.

B. C. Oltikar and Luis Ribes, On prosupersolvable groups ...

Brian Lee Peterson, Extensions of pro-affine algebraic groups ...

Thomas M. Phillips, Primitive extensions of Aronszajn spaces ...

Mehdi Radjabalipour, Equivalence of decomposable and 2-decomposable operators. .

M. Satyanarayana, Naturally totally ordered semigroups .

Fred Rex Sinal, A homeomorphism classification of wildly imbedded two-spheres in $S^{3}$

Hugh C. Williams, Some properties of a special set of recurring

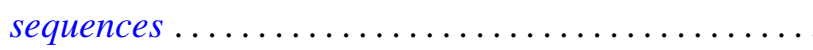

